\title{
Studies on mobile robots for all types of terrain
}

\author{
Robert Florian Pecie ${ }^{1}$, Mihai Olimpiu Tătar ${ }^{1 *}$, and Călin Rusu ${ }^{1}$ \\ ${ }^{1}$ Department of Mechatronics and Machine Dynamics, Technical University of Cluj-Napoca, Cluj- \\ Napoca, Romania
}

\begin{abstract}
In the first part of the paper, the authors present the characteristics of the robots for all types of terrain. In the second part, two categories of robots are proposed: a robot with hybrid locomotion system and a modular robot. For the last category, if different modules are combined, a family of modular robots adaptable to different types of terrain can be obtained. The solutions proposed by the authors allow the study of the mobility and adaptability of robots to different types of terrain.
\end{abstract}

\section{Introduction}

Robotics for difficult terrain is a rapidly evolving field of research. In this field, researchers make great efforts to achieve a higher level of autonomy.

The fields of application of these robots are scientific exploration of hostile environments such as deserts, volcanoes, the Arctic and search and rescue operations [1]. These robots can generally be equipped with hybrid locomotion systems or they can have a modular structure.

Mobile robots have recently been designed to move using a wide variety of locomotion systems, including legs, wheels, or tracks. Thus, the use of wheeled robots brings speed, efficiency and does not require complicated control algorithms, but has limited applicability to surfaces with small bumps.

Tracks allows movements at lower speeds but are more suitable for various types of terrain with small to medium bumps. The use of legs has a high adaptability to various environments but requires a more complex mechanical structure and complicated control algorithms.

In most cases, a single type of locomotion is not enough for all types of terrain, as there are complex operating environments that include steep bumps, ditches, and obstacles of various heights.

\section{Hybrid robots}

The three main categories of locomotion systems used on land, are based on wheels (W), tracks $(\mathrm{T})$ or legs $(\mathrm{L})$, and starting from them four categories of hybrid locomotion systems can be obtained: W-T, L-W, T-L, W-T-L [2], [3].

\footnotetext{
* Corresponding author: calin.rusu@mdm.utcluj.ro;
} 
The adaptability of a mobile robot to various environments can be solved by using a hybrid locomotion system that combines the main modes of travel thus bringing flexibility and efficiency, so that a multitude of applications can be made with the same mobile platform.

For a robot to be able to adapt to different types of terrain, two modes of adaptation are identified in the literature [4]:

- Active adaptation which requires an additional actuator to operate the locomotion system.

- Passive adaptation who does not require an additional device to operate the locomotion system, as it uses the effect of gravity to adapt to an obstacle course.

We mention that in the literature there is a great diversity of robots with this architecture, (robots that can adapt to different types of terrain) [5-8]. Some representative examples of hybrid robots for rough terrain with active and passive adaptation from the literature are presented in table 1.

Table 1. Examples of hybrid robots.

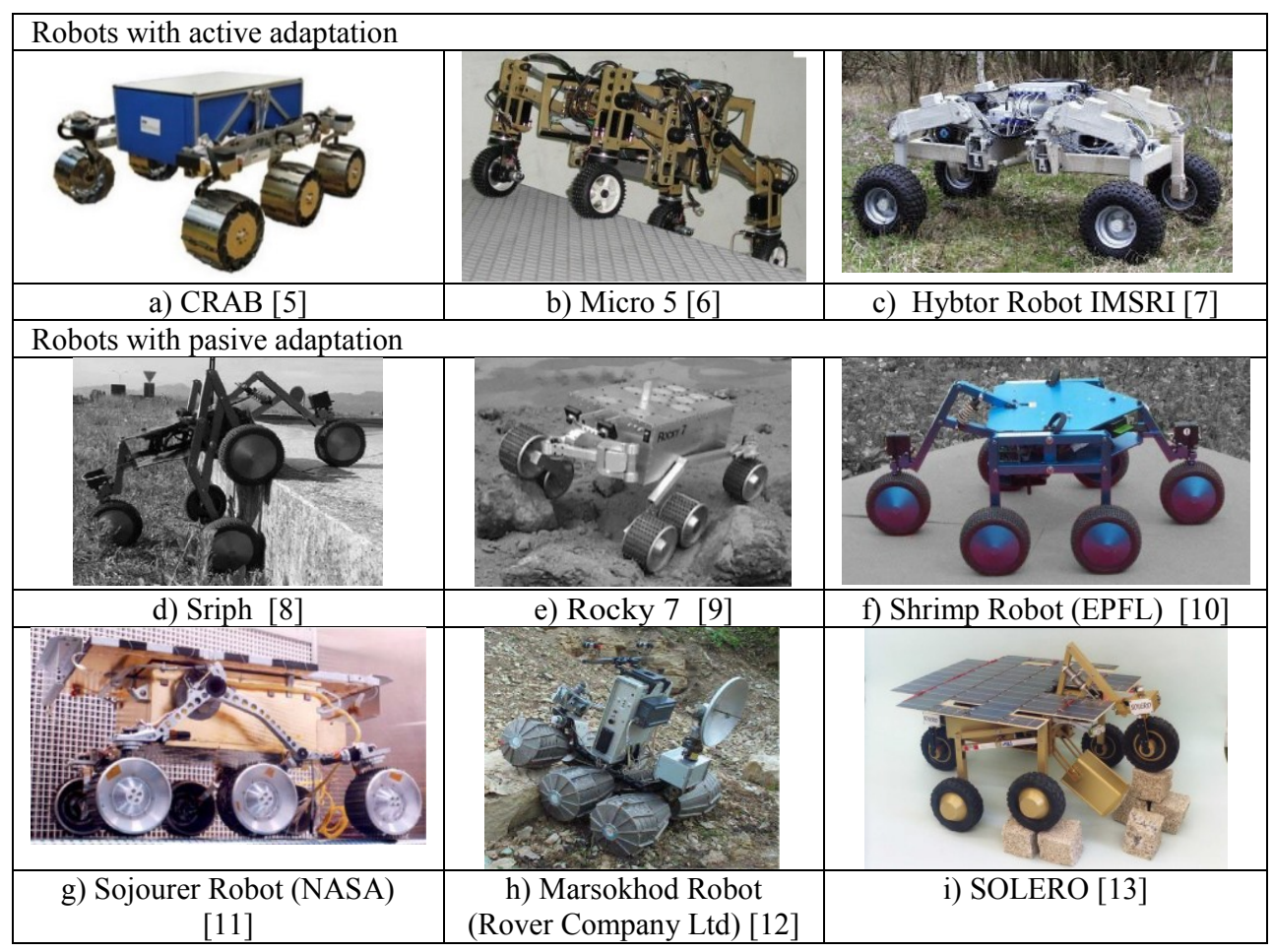

In the case of these robots, an identification possibility is represented by the use of the wheel formula (WF) which can be expressed as [13]:

$$
W F=n_{w} \cdot n_{a w} \cdot n_{\text {saw }}
$$

where: $n_{w}$ represents the represents the total number of wheels, $n_{a w}$ is the number of actuated wheels and $n_{\text {saw }}$ are the number of steered actuated wheels. If a robot has a walking mechanism, it is also included in the wheel formula separated by a plus $(+)$ sign.

There are several factors that must be considered when designing these robots. These include the size of the robot and its locomotion system, both depending on the robot's applications [14]. 


\section{Modular articulated robots (snake type robots)}

Recently, several achievements in the field of articulated modular robots (snake type), have been recorded in the literature [15], [16], [17], [20-21], [18]. This type of robots is composed of several modules, both active and passive. Depending on the locomotion system, these robots can generally be classified into modular crawler robots and crawler modular robots.

Another possibility of classification according to the mode of operation of the wheels/tracks and the joints that connect the robot modules is presented in $[15,16]$.

Thus, robots with active wheel / active crawler (AW/AT) and / or active joint (AJ) / passive joint (PJ) / can be identified. AW-PJ (Active-Wheel Passive-Joint articulated mobile robots) robots have high speed, mobility, and high durability on rough terrain, but can become blocked due to passive joints [15].

Snake-like robots take on the shape and form of a snake but use propulsive elements such as wheels and crawler for locomotion [25].

In [17] another classification is presented in which five categories of modular robots are listed (snake-inspired robot) without considering the articulation between the modules:

- robots with passive wheels,

- robots with active wheels,

- robots with active treads,

- robots based on undulation using vertical waves,

- robots based on undulation using linear expansion.

Although crawler robots have a better climbing ability than wheeled robots, they are slower, more voluminous and have a low ground clearance. It is also difficult to design a waterproof and dustproof system for crawler / crawler robots [16].

Therefore, the design of the robot on wheels is chosen because, in a rescue scenario, speed is of vital importance. Some representative examples of modular articulated robots for rough terrain in the literature are presented in Table 2.

Table 2. Examples of modular articulated robots

\begin{tabular}{|l|l|l|}
\hline a) KAIRO 3 [18]; AW-AJ type & b) Genbu 3 [19]; AW-PJ type & $\begin{array}{l}\text { ACM-R3 [20-21]; } \\
\text { PW-AJ type }\end{array}$ \\
\hline & & \\
\hline
\end{tabular}

\section{Proposed models}

Next, in this paragraph the authors proposals in this field, are presented. 


\subsection{First model}

The first proposed model is a robot for difficult terrain with hybrid wheel-leg locomotion system (Fig. 1). Current research shows that hybrid locomotion systems (L-W wheel-leg) are suitable when it is necessary to combine the speed and energy efficiency of wheels with the ability to climb and the operational flexibility of the legs [24].

The proposed robot has 6 legs each with a wheel at the end. It is equipped with active and passive suspension systems.

At the front, the robot has four legs equipped with active suspension (controlled by DC motors). The first pair of legs has the role of orienting the robot. The orientation of the legs is provided by two gearbox DC motors.

The back of the robot is composed of two legs. The suspension is passive using two shock absorbers. Their wheels are also driven by DC motors.

This type of robot has good handling on rough terrain. For this purpose, it uses several motors that are controlled by an electronic board. Powering the motors and the electronic board is done using batteries.

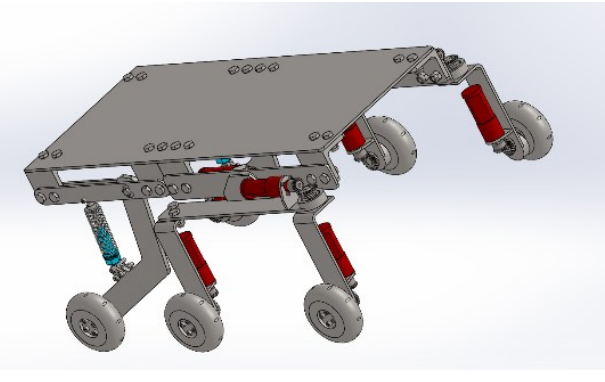

a)

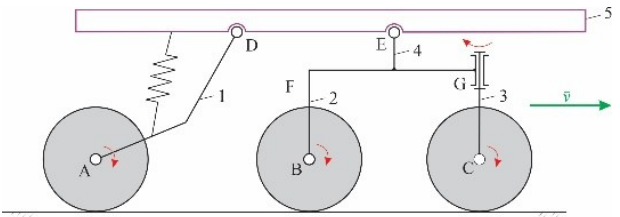

b)

Fig. 1. Robot with hybrid locomotive system a) 3D model b) Schematic representation.

Maneuverability on rough terrain is only possible if sensors are used to read the terrain to be traversed. In general, processing the data provided by the sensors and making decisions accordingly takes some time. There is also a time required for the structure to adapt to terrain variations. For these reasons, the robot can be quite slow.

When the robot encounters an obstacle, the front side tries to adapt to the terrain. In case of passing over a step the front of the robot can adapt to this situation due to the active suspension, and if it can no longer face the obstacle the back of the robot, with passive suspension and separately controlled wheels, can push the robot over the obstacle.

The main modules that ensure the control and operation of the robot are presented in the Fig. 2.

The central element of the robot's command and control system is an Arduino Due 32bit ARM microcontroller board. It controls the traction motors of the robot via three Cytron 10A 5-30V dual channel DC motor drivers. Each of these drivers provides bi-directional control for two brushed DC motor and supports both locked-antiphase and sign-magnitude PWM operation. The DC motors for steering and active suspension are equipped with incremental sensors and are controlled in a closed loop.

The control algorithms run in the Arduino microcontroller and the control signals are sent to the motors via two Cytron 3A 4-16V dual channel DC motor drivers. In order to detect obstacles and to be able to adapt to terrain variations, the robot is also equipped with various sensors (accelerometer, compass, ultrasonic sensors). Remote control and communication with the robot is provided by an $\mathrm{RC}$ transceiver. 


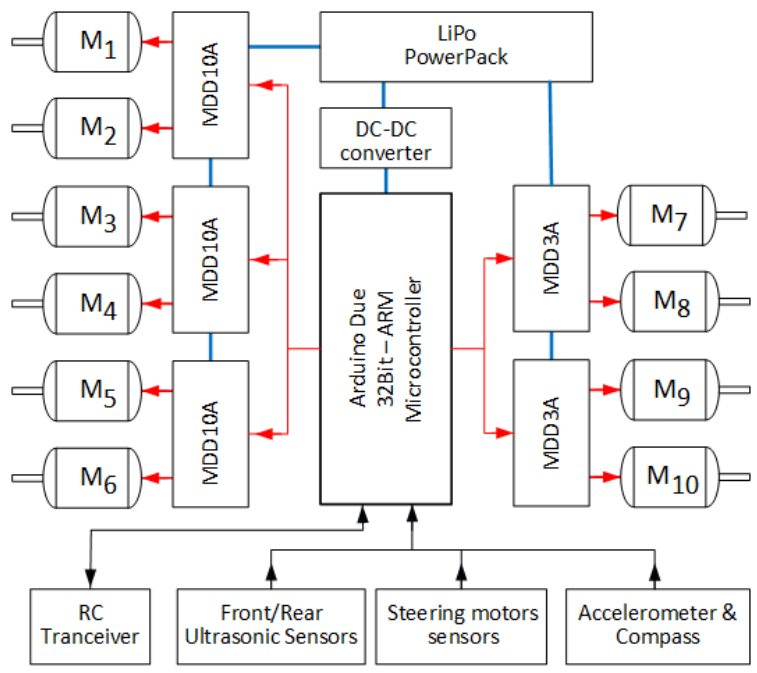

Fig. 2. Main modules used for robot control.

\subsection{The second model}

When operating on rough terrain, modular robots with wheels, legs or crawlers have more advantages than single-module (body) robots. First of all, the flexibility offered by their multiple modules (bodies) allows them to avoid many terrain deformations to which rigidbody robots are prone.

Also, through proper control of their various modules, these robots consisting of several modules can select the place they come in contact with the ground, thus reducing their vulnerability in case of traction problems. In this sense, the second model proposed by the authors is a modular articulated robot with wheels for difficult terrain that can be manufactured in different configurations. The modules of the proposed robot are of two types: motor modules (active) (Fig. 3 a) containing the drive motors and passive modules containing the electronic boards, etc. (Fig. 3 b). The propulsion of the robot is ensured by the motor modules that have in component two motor wheels driven independently.

The connection between these modules is realized through passive joints in this case, but constructive solutions of modular robots with active (operated) joints can be also designed. The proposed models are driven by servomotors and the power is ensured by an external voltage source through cables. It is also possible to use batteries that can be placed in passive modules. But this solution would increase the weight of the robot, which would increase the current required to drive the motors and decrease the life of the batteries.

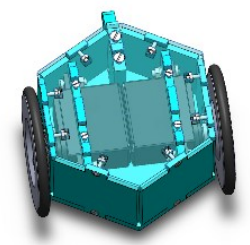

a)

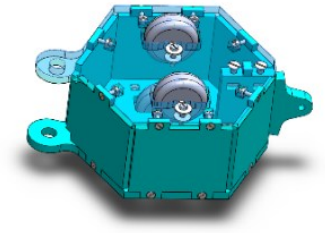

b)

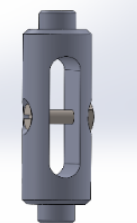

c)

Fig. 3. 3D modeling of the modular robot components a) driving mode b) passive mode c) coupling element. 
By combining the active and passive modules, a family of modular robots can be obtained, which will be presented below. The number of these modules can be chosen by the operator according to needs (use of the modular robot). We chose the active wheel as the propulsion device due to its mechanical robustness and simplicity and due to the fact that the locomotion capacity of the robot can be increased by inserting them. Using the modules shown in Figure 3, the following four possible configurations are described below.

\section{1st Configuration}

The first proposed configuration (Fig. 4) consists of a driving module and several passive modules connected by rotating joints (trailer modular robot). Due to its particular structure, it is recommended to be used on flat surfaces.

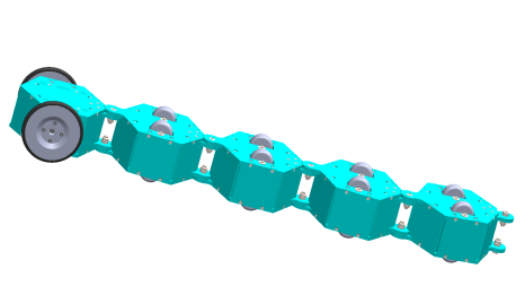

a)

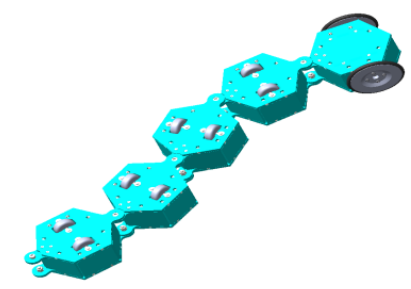

b)

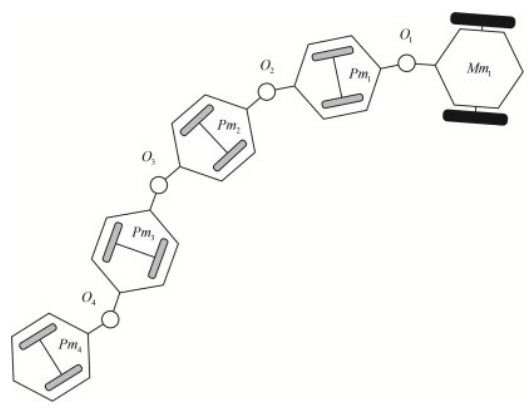

c)

Fig. 4. Modular robot v1: a), b) 3D modeling c) schematic structure $\left(P_{m}\right.$ passive module, $M_{m}$ driving module).

\section{2nd Configuration}

This configuration presented in Figure 5. consists of a driving module and several passive modules connected by universal joints. This solution can overcome small obstacles.

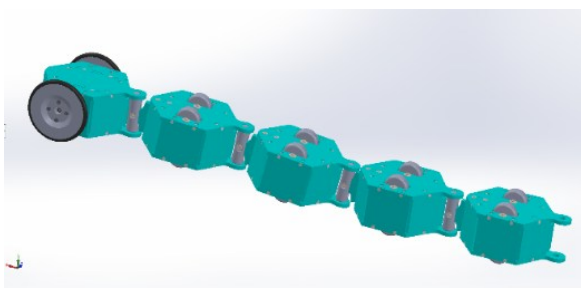

a)

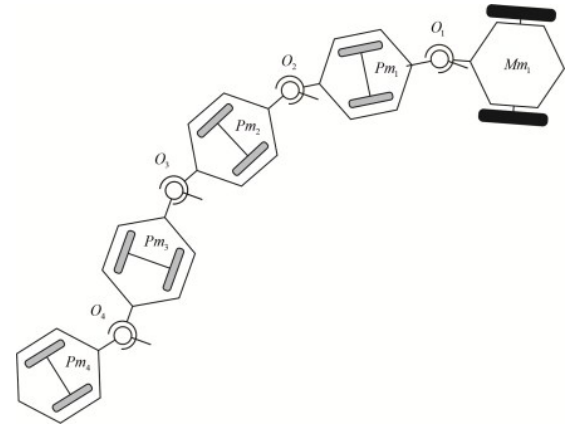

b)

Fig. 5. Modular robot v2: a) 3D modeling b) schematic representation $\left(P_{m}\right.$ passive module, $M_{m}$ driving module). 


\section{3rd Configuration}

For a better mobility and for the increase of the traction force, the solution presented in Fig. 6 can be used. In this case, universal joints are used between the modules. This solution uses one driving module after each passive module. The configuration shown in Figure 6 has three active and two passive modules.

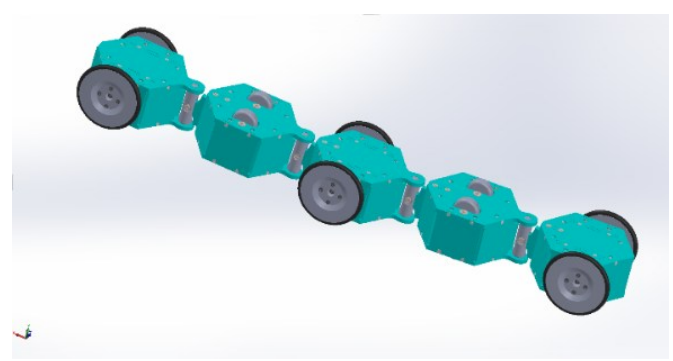

a)

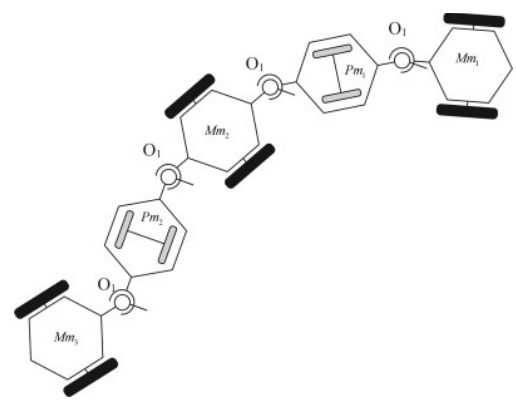

b)

Fig. 6. Modular robot v3: a) 3D modeling b) schematic representation $\left(P_{m}\right.$ passive module, $M_{m}$ driving module).

\section{4th Configuration}

In the configuration shown in Fig. 6 the driving modules are connected through universal joints. This solution can overcome much greater obstacles compared to the first one previously presented.

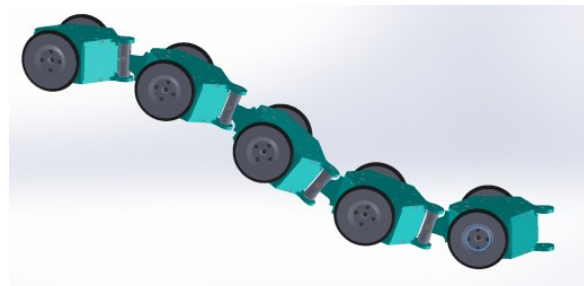

a)

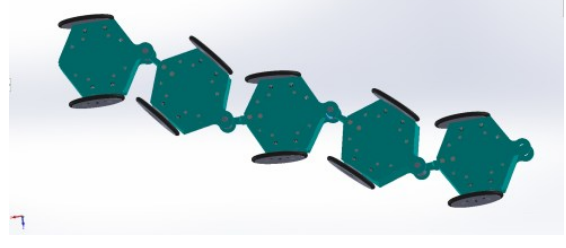

b)

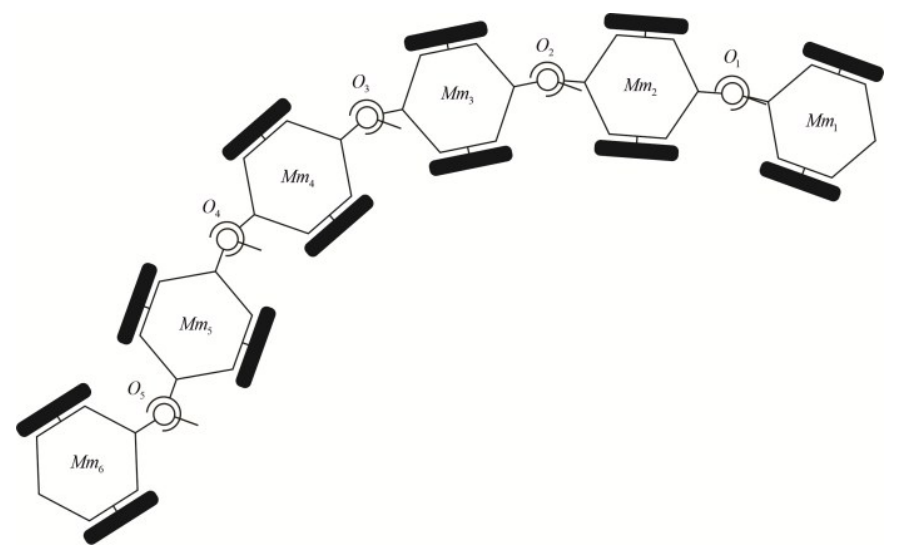

c)

Fig. 7. Modular robot v4: a), b) 3D modeling c) schematic representation $\left(M_{m}\right.$ driving module). 


\section{Mathematical model}

In the case of a modular system with only one traction module (Fig. 4) the kinematic modeling starts from the kinematic model of a car with two degrees of freedom pulling $n$ trailers [25], [26].

We denote with $\left(x_{i}, y_{i}, \theta_{i}\right), \mathrm{i}=0,2, \mathrm{n}$ the robot configuration, where $\left(x_{i}, y_{i}\right)$ are the coordinates of the point in the middle of module axis $i, \theta_{i}$ is the orientation angle of the module $i$ with respect to the $x$ axis, $v_{i}$ represents the linear velocity of module $i$ and $\omega_{i}=\dot{\theta}_{i}$ is the angular velocity of the module $i$.

The coordinates of the point in the middle of the axis between the two wheels of the engine mode satisfy the relation:

$$
\begin{aligned}
\dot{x}_{0}= & v_{0} \cos \theta_{0} \\
& \dot{y}_{0}=v_{0} \sin \theta_{0}
\end{aligned}
$$

or written in matrix form, the relation become:

$$
\left[\begin{array}{c}
\dot{x}_{0} \\
\dot{y}_{0}
\end{array}\right]=v_{0}\left[\begin{array}{c}
\cos \theta_{0} \\
\sin \theta_{0}
\end{array}\right]
$$

where $v_{o}$ is the linear velocity and $\omega_{0}$ is the angular velocity of the motor module (are the inputs to the system).

For the robot with $n$ module, starting from the relations:

$$
\begin{gathered}
x_{n-1}=x_{n}+d_{n} \cos \theta_{n}+d_{n-1} \cos \theta_{n-1} \\
x_{n-1}=x_{n}+d_{n} \sin \theta_{n}+d_{n-1} \sin \theta_{n-1}
\end{gathered}
$$

and their derivatives:

$$
\begin{gathered}
v_{n-1}=v_{n}-d_{n} \dot{\theta}_{n} \sin \theta_{n}-d_{n-1} \dot{\theta}_{n-1} \sin \theta_{n-1} \\
v_{n-1}=v_{n}+d_{n} \dot{\theta}_{n} \cos \theta_{n}+d_{n-1} \dot{\theta}_{n-1} \cos \theta_{n-1}
\end{gathered}
$$

and the non-holonomic constraint relationship:

$$
x_{n} \sin \theta_{n}-y_{n} \cos \theta_{n}=0
$$

the following recursive equations can be determined in the case of the proposed modular robot:

$$
\begin{aligned}
& v_{i}=\cos \left(\theta_{i-1}-\theta_{i}\right) v_{i-1}+d_{i-1} \dot{\theta}_{i-1} \sin \left(\theta_{i-1}-\theta_{i}\right) \\
& \dot{\theta}_{i}=\frac{1}{d_{i}}\left[\sin \left(\theta_{i-1}-\theta_{i}\right) v_{i-1}-d_{i-1} \dot{\theta}_{i-1} \cos \left(\theta_{i-1}-\theta_{i}\right)\right] \\
& {\left[\begin{array}{l}
x_{i} \\
y_{i}
\end{array}\right]=\left[\begin{array}{l}
x_{i-1} \\
y_{i-1}
\end{array}\right]-d_{i-1}\left[\begin{array}{c}
\cos \theta_{i-1} \\
\sin \theta_{i-1}
\end{array}\right]-d_{i}\left[\begin{array}{c}
\cos \theta_{i-1} \\
\sin \theta_{i-1}
\end{array}\right], i=1,2 \ldots n}
\end{aligned}
$$


where $d_{i}=O_{i} O_{i-1}$ represents the distance between joints $O_{i}$ and $O_{i-1}$. The distances between the connections between the modules and the centers of these modules are equal.

In Fig. 8 the kinematic structure of a modular robot with $n$ modules with variable configurations, the control inputs and kinematic parameters are presented

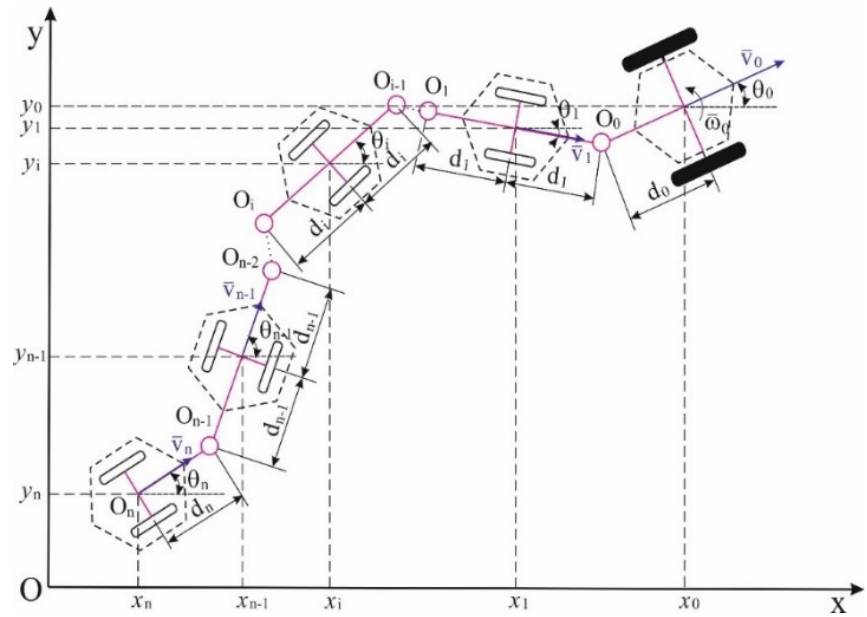

Fig. 8 Kinematic structure of the modular robot with $n$ modules

Equations (1), (6), (7), (8) represents the kinematic model of the modular robot with $n$ modules. This model can be easily applied for example to the robot presented in Figure 4 in which case $\mathrm{n}=4, i=1 \ldots 4$.

\section{Conclusions}

In this paper, a brief study of the main types of mobile robots for rough terrain was proposed. The authors presented in more detail two configurations of mobile robots that will be the subject of future research. The solutions proposed by the authors allow the study of the mobility and adaptability of robots to different types of terrain.

The first proposed robot falls into the category of hybrid robots with leg-wheel locomotion system.

The second robot is a modular robot that can be included in the category of snake robots. that can be easily manufactured with a laser cutting plotter and obtain fast experimental results These robots due to their advantages have a great potential especially in the field of search and rescue. At the end of the paper, the kinematic model for a modular trailer robot is presented.

In the next stage of the research, the authors aim to build, and test experimental models based on the two types of robots presented in the paper. Special attention will be paid to the second model of the robot which can be made quite easily by laser cutting methods. In this way it will be possible to validate the proposed mathematical model and possibly make some improvements.

\section{References}

1. L. Pierre, $3 D$ position tracking for all-terrain robots (EPFL, Lausanne, 2005)

2. L. Bruzzone, G. Quaglia, Mechanical sciences, 3, 49 (2012)

3. M. O. Tătar, P. Barbu, E Teuţan, AQTR (2018) 
4. J. Trevelyan, S. C. Kang, W. R. Hamel, Springer Handbook of Robotics (Springer-Verlag Berlin Heidelberg 2008)

5. A. Krebs, T. Thueer, E. Carrasco, R. Oung, R. Siegwart, i-SAIRAS'08, (2008)

6. F. B. Amar, Ch Grand, G. Besseron, F. Plumet, ASTRA (2004)

7. I. Leppänen, S. Salmi, A. Halme, CLAWAR (1998)

8. R. Siegwart, P. Lamon, T. Estier, M. Lauria, R. Piguet, Rob. Auton. Syst. 40, 151 (2002)

9. R. Volpe, J. Balaram, T. Ohm, R. Ivlev, Adv. Robotics, 11, 341 (1996)

10. S. Michaud, A. Schneider, R. Bertrand, P, Lamon, R, Siegwart, M, van Winnendael, A. Schiele, ASTRA SOLERO (2002)

11. R. Volpe, J. Balaram, T. Ohm, T. Ivlev, IROS (1996)

12. A. Kemurdjian, V. Gromov, V. Mishkinyuk, V. Kucherenko, P. Sologub, ICRA (1992)

13. A. Seeni, B. Schafer, B. Rebele, N. Tolyarenko, Aerosp. Conf. Proc. (2008)

14. S. Lim, T. Jason, WSEAS Transactions on systems, 14, 11 (2015)

15. H. Kimura, S. Hirose, K. Shimizu, ICRA (2004)

16. K. Suzuki, A. Nakano, G. Endo, Hirose, IROS (2012)

17. J. K. Hopkins, B. W. Spranklin, S. K. Gupta, Bioinspir.Biomim., 4, 189 (2009)

18. L. Pfotzer, S. Klemm, A. Rönnau, J. M. Zöllner, R. Dillmann, Rob. Auton. Syst. 89, 123 (2017)

19. H. Kimura, S. Hirose, ICRA (2002)

20. M. Mori, S. Hirose, IROS (2001)

21. M. Neumann, T. Predki, L. Heckes, P. Labenda, Ind. Rob., 40, 246 (2012)

22. G. Granosik, J. Borenstein, IEEE/ASME Trans. Mechatron., 10, 473 (2005)

23. P. Labenda, T. Sadek, L. Heckes, IFAC Proc. Vol., 43, 739 (2010)

24. L. Bruzzone, P. Fanghella, Ind. Rob., 41, 26 (2014)

25. M. Park, W. Chung, M. Kim, J. Song, Control ICRA (2004)

26. J. Yuan, Y. Huang, F. Sun, Design ROBIO (2004) 